\title{
Regeneração de raízes e plântulas de sementes de Eugenia spp. (Myrtaceae) de diferentes estádios de maturação
}

\author{
(D) Liliana Ferreira Delgado e e iD Claudio José Barbedo ${ }^{2,3}$
}

Recebido: 14.01.2020; aceito: 29.06.2020

Como citar: Delgado, L.F. \& Barbedo, C.J. 2020. Regeneração de raízes e plântulas de sementes de Eugenia spp. (Myrtaceae) de diferentes estádios de maturação. Hoehnea 47: e042020. http://dx.doi.org/10.1590/2236-8906-04/2020.

\begin{abstract}
Root and seedling regeneration of Eugenia spp. (Myrtaceae) of different maturity stages). The regenerative capacity of Eugenia seeds is present from the still immature seeds to those that have already started germination. However, little is known about the limits of this capacity at the different stages of seed development. In this work we studied the physical and physiological modifications of fruits and seeds of five species of Eugenia during their formation and maturation, as well as the development of the seedlings after removal part of the seed reserves. The results showed that there is no developmental pattern for the different species of the genus, but the seeds of all species have high capacity to producing normal seedlings even from immature fruits and after some reserve material lost.

Keywords: cutting seeds, physiological maturity, recalcitrant seeds
\end{abstract}

RESUMO -(Regeneração de raízes e plântulas de sementes de Eugenia spp. (Myrtaceae) de diferentes estádios de maturação). A capacidade regenerativa das sementes de Eugenia está presente desde as sementes ainda imaturas até as que já iniciaram a germinação. No entanto, pouco se sabe sobre os limites dessa capacidade em vários estágios da formação de sementes. Neste trabalho procurou-se associar as modificações físicas e fisiológicas de frutos e sementes de cinco espécies de Eugenia durante sua formação e maturação, bem como o potencial de produção de raízes e plântulas após a remoção de parte de suas reservas. Os resultados mostraram que não há padrão de desenvolvimento para as diferentes espécies do gênero, mas as sementes de todas as espécies têm alta capacidade de germinar e produzir plântulas normais, mesmo quando obtidas de frutos imaturos e com perda de material de reserva da semente.

Palavras-chave: fracionamento de sementes, maturidade fisiológica, sementes recalcitrantes

\section{Introdução}

Diversos estudos sobre fracionamento de sementes de Eugenia demonstraram seu elevado potencial regenerativo (Anjos \& Ferraz 1999, Silva et al. 2005, Amador \& Barbedo 2011, Delgado et al. 2010, Prataviera et al. 2015, Alonso et al. 2019). Essa característica, além de possivelmente conferir vantagem seletiva para essas espécies, especialmente quando aliada a outras características como sua adaptação a déficits hídricos (Inocente \& Barbedo 2019), poderia ser aproveitada no planejamento da produção de mudas, maximizando o potencial produtivo de lotes de sementes e auxiliando nos processos de conservação das espécies, principalmente para as que se encontram em risco de extinção (Teixeira \& Barbedo 2012).

Os estudos acerca dessa habilidade em formar novas raízes (sem desenvolvimento de parte aérea, doravante denominado apenas formação de raízes) ou mesmo plântulas inteiras (quando, além de raízes, há desenvolvimento de parte aérea completa, doravante denominada apenas formação de plântulas) a partir do tecido cotiledonar (Delgado 2010) tem avançado em diversos aspectos. Há, por exemplo, evidente controle interno para que as reservas da semente não sejam utilizadas em germinações simultâneas e, dessa forma,

1. Escola Municipal de Ensino Fundamental Raul de Leoni, Secretaria Municipal de Educação de São Paulo, Avenida José da Rocha Viana, 44, Vila Pedra Branca, 02635-090 São Paulo, SP, Brasil

2. Instituto de Botânica, Núcleo de Pesquisa em Sementes, Avenida Miguel Stéfano, 3687, 04301-012 São Paulo, SP, Brasil

3. Autor para correspondência: cjbarbedo@yahoo.com.br 
quando uma germinação se inicia há inibição da formação de novas raízes na mesma semente, como demonstrado em Eugenia uniflora L. e E. brasiliensis Lam. (Delgado $\&$ Barbedo 2011, Amador \& Barbedo 2015). A quantidade de tecido de reserva parece também definir o sucesso da formação de novas plântulas, muitas vezes obtendo-se somente novas raízes (Prataviera et al. 2015). A cada perda de raízes e/ou plântulas, as novas que se formam necessitam tempos cada vez maiores, o que permite que a espécie tenha indivíduos capazes de colonizar novas áreas por um longo período (Alonso \& Barbedo 2020). Contudo, alguns aspectos ainda são pouco estudados, como o período compreendido por essa capacidade, desde a formação até a germinação das sementes. A capacidade regenerativa deve ser analisada não apenas em uma das fases do processo de maturação uma vez que, por serem sementes do grupo das recalcitrantes (Delgado \& Barbedo 2007), as sementes das espécies de Eugenia não demonstram evidente maturidade fisiológica, mas um continuum entre a formação da semente e sua germinação (Barbedo et al. 2013). Portanto, o desenvolvimento e a maturação dessas sementes em relação à capacidade de regeneração são aspectos importantes a serem considerados.

$\mathrm{O}$ desenvolvimento da maioria das sementes pode ser dividido em três fases, sendo a primeira caracterizada pelo crescimento inicial, aumento rápido no peso fresco e no conteúdo de água; em um segundo momento a semente aumenta de tamanho devido a expansão das células e a deposição de reservas e finalmente, para as sementes de outro grupo, o das ortodoxas, o desenvolvimento termina com uma fase pré-programada de secagem ou dessecação (Barbedo \& Santos Junior 2018). Dados relativos ao comportamento das sementes de Eugenia indicam que são sensíveis à desidratação, com baixa longevidade (Delgado \& Barbedo 2007), apresentando teor de água em torno de $40-70 \%$ quando dispersas (Anjos \& Ferraz 1999, Andrade \& Ferreira 2000, Andrade et al. 2003, Santos et al. 2004, Delgado \& Barbedo 2007). Esse elevado teor de água no momento da dispersão as coloca em condições já próximas à germinação, diferenciando-se das ortodoxas que são dispersas com teor de água bem mais baixo, frequentemente abaixo de $20 \%$.

Durante a evolução das espécies tropicais, algumas adquiriram a característica de sincronizar a maturidade fisiológica de suas sementes com mudanças nas cores dos frutos, sugerindo uma ligação com a síndrome de dispersão, uma vez que poderia atrair potenciais animais, tanto polinizadores quanto dispersores, influenciando a qualidade das sementes colhidas (Guardia et al. 2018, Silva et al. 2018, Fidalgo et al. 2019). Para frutos carnosos, o problema maior seria o conhecimento da época em que ocorre a maturação fisiológica das sementes já que nem sempre há necessidade de esperar a completa maturação "visual" do fruto. A mudança de cor do fruto nem sempre é eficiente para a avaliação da maturidade fisiológica de sementes, não sendo indicativo de maturidade para sementes de algumas espécies (Lamarca et al. 2013, 2016). Tal fato sugere um descompasso entre a maturação dos frutos e a das sementes sendo necessário, portanto, um acompanhamento em intervalos de tempo mais curtos que visem avaliar outras características além da coloração do fruto. Embora a capacidade regenerativa das sementes de Eugenia esteja presente durante um longo período, desde as sementes ainda imaturas até as que já iniciaram a germinação (Teixeira \& Barbedo 2012), pouco se sabe sobre os limites dessa capacidade em diversas fases da formação das sementes.

A associação entre as características externas visuais dos frutos, as físicas e fisiológicas de suas sementes e, particularmente para as sementes de Eugenia, as mudanças na eficiência da regeneração de raiz e parte aérea após remoção de parte do tecido de reserva nas diferentes fases da maturação são essenciais para o planejamento e manejo de lotes de sementes. Dessa forma, as hipóteses deste trabalho foram que 1) pode haver sincronicidade entre o processo de maturação de frutos e sementes e 2) sementes de espécies de Eugenia podem apresentar capacidade regenerativa desde fases mais imaturas.

\section{Material e métodos}

Obtenção do material vegetal - Os frutos de Eugenia uniflora L., E. brasiliensis Lam., E. pyriformis Cambess., E. involucrata DC. e E. cerasiflora Miq. (Myrtaceae), foram coletados em períodos distintos durante a maturação, de setembro a dezembro de 2014. Inicialmente, flores foram marcadas no momento da antese para referenciar os frutos característicos de cada idade em cada coleta. Baseando-se nas características dos frutos oriundos das flores marcadas, em cada coleta foram colhidos cerca de 150-200 frutos mais representativos da época, descartando-se os que se encontravam fora do padrão. Foram realizadas cinco coletas (para as quatro primeiras espécies) ou seis (para E. cerasiflora), obtendo-se os estádios de I a VI, sendo o primeiro somente para E. cerasiflora (figura 1). Em seguida, foram imediatamente levados ao laboratório para extração das sementes (figura 1). As coletas de frutos de E. uniflora foram realizadas em cinco árvores de propriedades privadas na Zona Norte da cidade de São Paulo, SP, Brasil; (2327'S e $46^{\circ} 36^{\prime} \mathrm{W}$ ) a das demais espécies foram realizadas em 2 a 3 árvores do Jardim Botânico de São Paulo, em São Paulo, SP, Brasil ( $\left.23^{\circ} 38^{\prime} \mathrm{S}, 46^{\circ} 37^{\prime} \mathrm{W}\right)$.

As sementes foram extraídas manualmente dos frutos com auxílio de peneira, em água corrente, sendo imediatamente retirado o excesso de umidade destas com papel filtro; as sementes dos frutos mais imaturos, em razão destes apresentarem polpa mais firme, foram extraídas manualmente com auxílio de bisturi. Logo após a extração, as sementes foram avaliadas quanto ao teor de água, conteúdo de massa seca e germinação, para caracterização inicial do lote.

Avaliações físicas e fisiológicas - O teor de água e o conteúdo de massa seca das sementes foram quantificados 

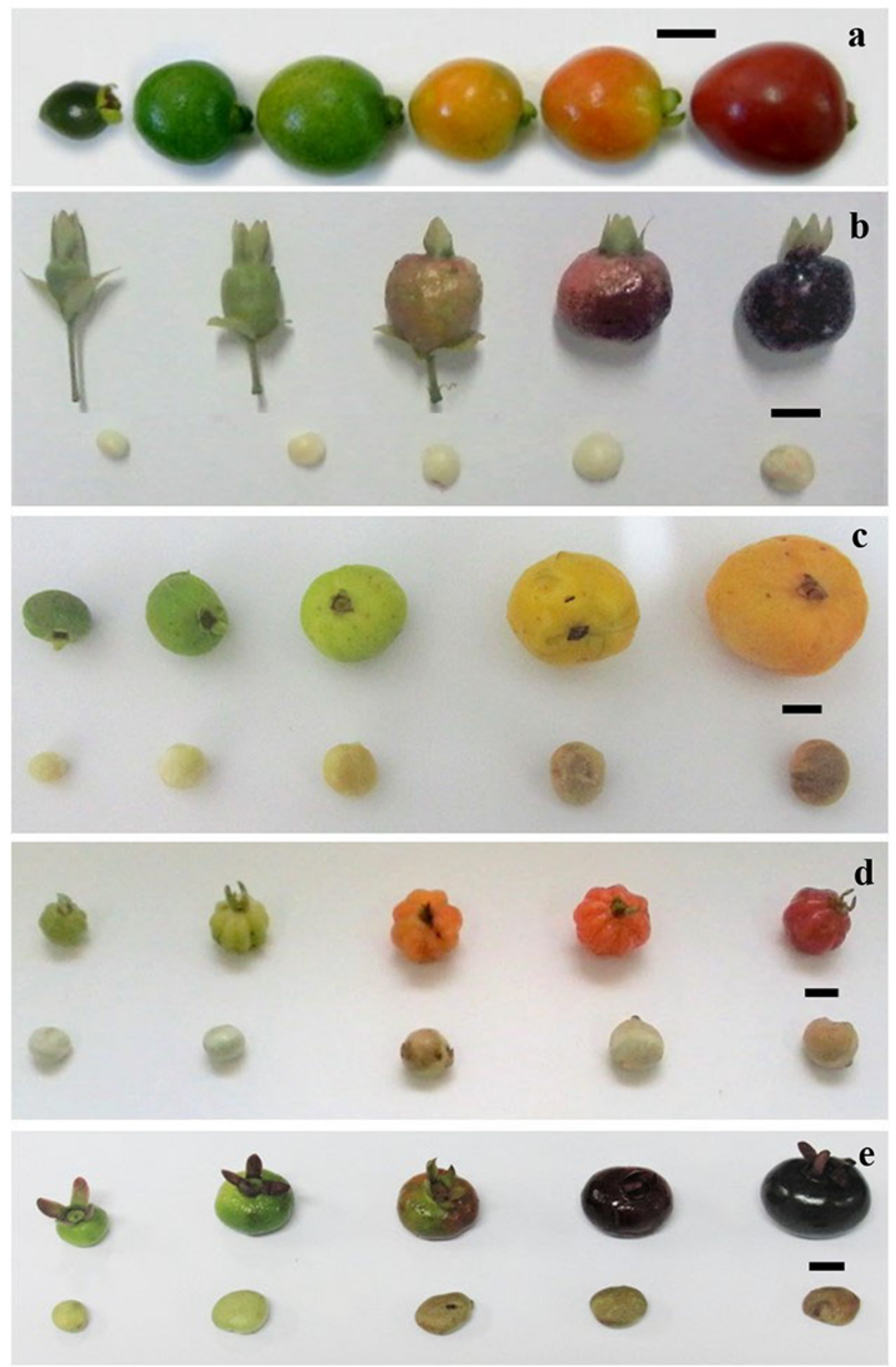

Figura 1. Estádios de maturação de frutos e suas respectivas sementes de espécies de Eugenia. a. Eugenia cerasiflora Miq., apenas frutos da esquerda para a direita, estádios de I a VI; b. E. involucrata DC. frutos e sementes dos estádios de II a VI; c. E. pyriformis Cambess., estádios de II a VI; d. E. uniflora L., estádios de II a VI; e. E. brasiliensis Lam., estádios de II a VI. Escala $=1 \mathrm{~cm}$.

Figure 1. Fruit maturity stages and their seeds from Eugenia species. a. Eugenia cerasiflora Miq., only fruits, from left to right stages I to VI. b. E. involucrata DC., fruits and seeds from stages II to VI. c. E. pyriformis Cambess., stages II to VI. d. E. uniflora L., stages II to VI. e. E. brasiliensis Lam.), stages II to VI. Scale $=1 \mathrm{~cm}$. 
conforme descrito nas Regras para Análise de Sementes (Brasil 2009), pelo método de estufa a $103 \pm 3{ }^{\circ} \mathrm{C}$, durante 17 horas, utilizando-se quatro repetições de cinco sementes cada, sendo expressos, respectivamente, em porcentagem (em base úmida) e mg semente ${ }^{-1}$.

$\mathrm{O}$ teste de germinação foi realizado em germinadores MA400 (Marconi, Piracicaba), com umidade relativa do ar de $100 \%$, mantida por circulação interna de água, regulados para a temperatura constante de $25^{\circ} \mathrm{C}$ e luz contínua. As sementes foram acondicionadas em gerbox, contendo papel tipo germitest, com duas folhas para a base e uma para cobertura (Brasil 2009), pré-umedecidas, utilizando-se quatro repetições de 10 sementes. Neste teste, as avaliações foram realizadas a cada sete dias, sendo computadas as sementes germinadas (protrusão de raíz primária com pelo menos $5 \mathrm{~mm}$ ) e as que resultaram no desenvolvimento de plântulas normais (plântulas com sistema radicular e eófilos desenvolvidos e sem defeitos aparentes, conforme Delgado \& Barbedo 2007), para os cálculos, respectivamente, de germinação e de desenvolvimento de plântulas normais.

Avaliação da capacidade em produzir raízes e plântulas após fracionamento - Para os estudos de regeneração de sementes em diferentes estádios de maturação, foram realizadas seções transversais ao meio, sendo o corte efetuado de tal forma que apenas uma das frações permanecesse com a cicatriz placentária (hilo). As porcentagens de germinação e de desenvolvimento de plântulas normais, para as sementes fracionadas, foram calculadas considerando-se somente a fração que continha o hilo. Contudo, para estas sementes fracionadas foram calculadas, também, as porcentagens de germinação e de desenvolvimento de plântulas normais somando-se as duas frações da mesma semente, conforme descrito em Silva et al. (2003) e Alonso et al. (2019).

Delineamento experimental e análise dos dados - Todos os experimentos tiveram delineamento inteiramente casualizado, considerando-se os estádios de maturação como tratamentos. Os resultados obtidos foram submetidos à análise de variância pelo teste $\mathrm{F}$ ao nível de $5 \%$ de probabilidade, dentro de cada espécie estudada e isoladamente para sementes íntegras ou fracionadas. As médias foram comparadas entre si pelo teste de Tukey ao nível de 5\% de probabilidade.

\section{Resultados e Discussão}

Os valores obtidos para teor de água de sementes das diferentes espécies de Eugenia demonstram que, como proposto por Barbedo et al. (2013) e Barbedo (2018), o processo de maturação de sementes sensíveis à dessecação (recalcitrantes) não segue os modelos clássicos descritos para as tolerantes (ortodoxas). Segundo esses modelos, durante a maturação o teor de água das sementes, inicialmente bastante elevado, vai diminuindo à medida em que a massa seca vai sendo acumulada até que atinjam cerca de 35-50\% (Marcos Filho 2015), chegando a valores frequentemente inferiores a 30\% quando são dispersas. Das cinco espécies estudadas neste trabalho, apenas uma chegou a valores próximos àqueles ( $E$. brasiliensis, com cerca de $50 \%$, figura 2$)$, as demais permanecendo com valores de 55 a $70 \%$ mesmo no último estádio de maturação, ou seja, em vias de serem dispersas (figura 2). O acúmulo de massa seca, por sua vez, nem sempre foi acompanhado de redução no teor de água, como verificado para E. pyriformis, em que o maior aumento na massa seca, do estádio III para o IV (figura 3 c), não foi acompanhado de mudança no teor de água (figura 2 c), e em E. uniflora, em que o aumento na massa seca de III para V (figura $3 \mathrm{~d}$ ) também não teve equivalente mudança no teor de água (figura $2 \mathrm{~d}$ ).

Segundo Marcos Filho (2015) o máximo acúmulo de massa seca, o baixo teor de água e a elevada capacidade germinativa, frequentemente e eficientemente utilizados para identificar a maturidade de sementes ortodoxas, não apresentam mesma eficiência para sementes recalcitrantes, uma vez que não há um ponto definido para o término da fase de maturação e correspondente início da germinação. Danner et al. (2010) observou, para sementes de E. uniflora, E. involucrata e E. pyriformis, lento acúmulo inicial da massa seca e grande aumento ao final da maturação dos frutos. No presente trabalho, isso foi mais evidente para sementes de E. cerasiflora (figura 3 a) e $E$. involucrata, mas não para $E$. uniflora e E. pyriformis (figura $3 \mathrm{~b}$ e c). Segundo Barbedo (2018), as condições ambientais nas quais as sementes são formadas podem influenciar o investimento nas diferentes características das sementes, ora beneficiando, por exemplo, a precocidade da germinação, ora o acúmulo de reservas. Portanto, as características de cada estádio de maturação dos frutos e das sementes podem ser alteradas conforme as condições climáticas nas quais as sementes são formadas e, portanto, os resultados em cada ano podem ser diferentes. Ainda assim, muitos estudos feitos com maturação de sementes de diversas espécies apontam o ponto de máximo de matéria seca como o melhor e mais seguro indicativo de que as sementes atingiram a maturidade fisiológica (Araújo et al. 2006), o que evidentemente não é o caso das sementes de Eugenia estudadas neste trabalho.

Outra característica interessante, hipotetizada por Barbedo (2018), é o investimento precoce em capacidade germinativa, comum em sementes recalcitrantes. Em todas as espécies estudadas a máxima germinação ocorreu já no estádio IV (ou antes, para algumas), com valores superiores a 90\% (figura 4), ou seja, três estádios antes do final, quando os frutos sequer apresentavam sua coloração final. Nesse estádio, inclusive, somente $E$. cerasiflora ainda não havia atingido os maiores valores de desenvolvimento de plântulas normais (figura 5), o que pode ser em função de que esta é, das cinco espécies, a menos domesticada. Conforme descrito por Lamarca et al. (2013) para E. pyriformis, a semente atinge sua máxima qualidade em momento anterior ao máximo amadurecimento dos frutos e, portanto, o critério de colheita de frutos maduros para obtenção da máxima qualidade de sementes não pode 

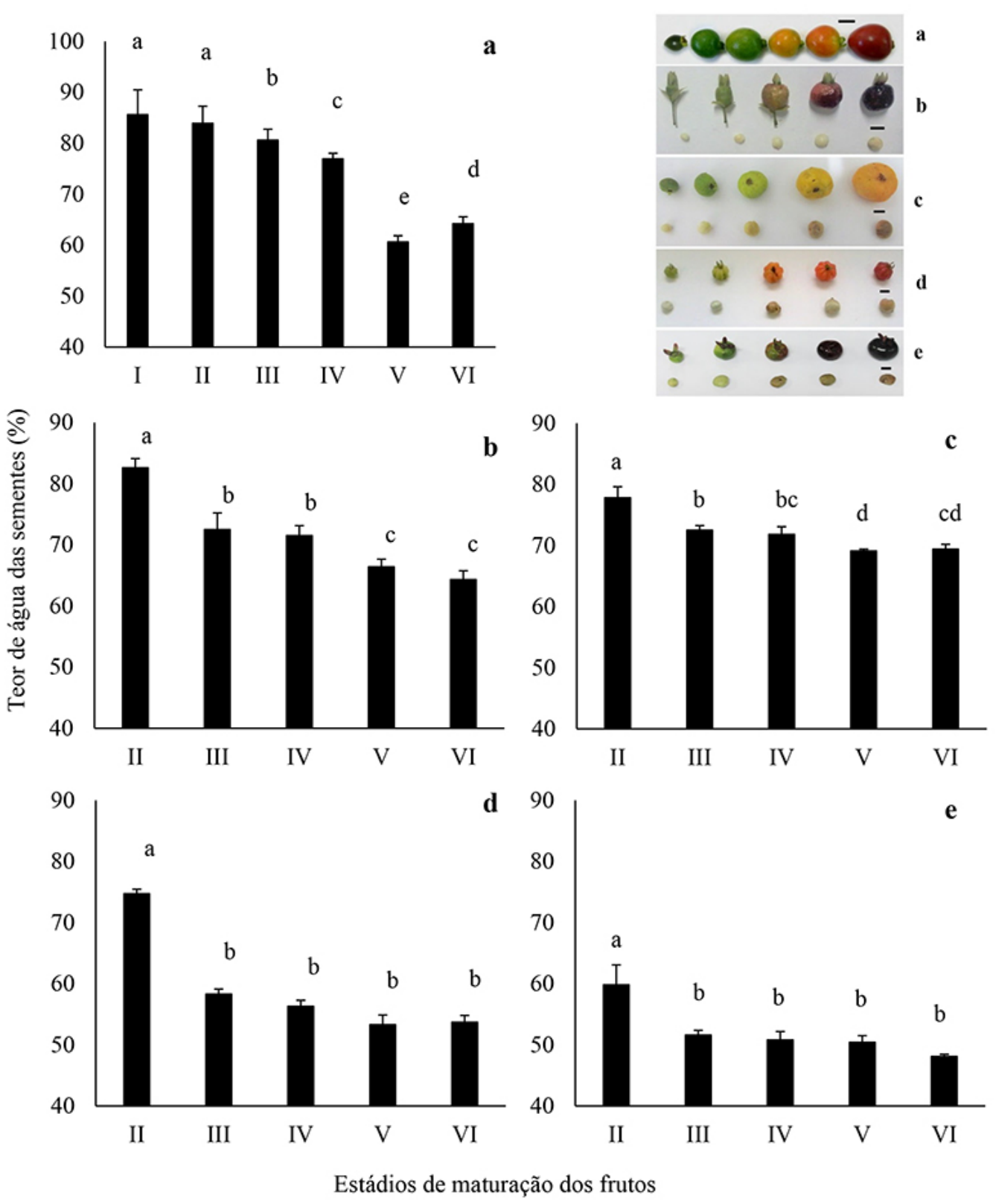

Figura 2. Teor de água de sementes de espécies de Eugenia, em função do estádio de maturação dos frutos (I a VI). a. Eugenia cerasiflora Miq. b. E. involucrata DC. c. E. pyriformis Cambess. d. E. uniflora L. e. E. brasiliensis Lam.). Médias seguidas pela mesma letra não diferem entre si pelo teste de Tukey (5\%).

Figure 2. Water content (\%) of seeds of Eugenia species, according to the fruit maturity stages (I to VI). a. Eugenia cerasiflora Miq. b. E. involucrata DC. c. E. pyriformis Cambess. d. E. uniflora L. e. E. brasiliensis Lam.). Means followed by the same letter do not differ by Tukey's test (5\%).

ser aplicado para E. pyriformis. Pelos resultados do presente trabalho, isso também poderia ser dito para outras espécies de Eugenia, como E. uniflora e E. brasiliensis. Nesta última, as sementes poderiam ser consideradas maduras já no estádio IV, quando já haviam atingido o maior valor de massa seca, o menor de teor de água e os mais elevados para germinação e desenvolvimento de plântulas normais (figuras $2 \mathrm{e}, 3 \mathrm{e}, 4 \mathrm{e}$ e 5 e). Curiosamente, a coloração predominante dos frutos, nesse estádio, era verde, bem diferente dos frutos maduros do estádio VI (figura 1). E em E. uniflora, as sementes poderiam ser consideradas maduras já entre os estádios IV e V, quando a cor dos frutos está próxima à dos maduros, mas ainda com tonalidade bem mais clara (figura 1). Ávila et al. (2009), em estudo de sementes de E. uniflora, concluiram que a coloração dos frutos é um parâmetro eficaz para a identificação do ponto de maturidade fisiológica desta espécie. O sincronismo da 


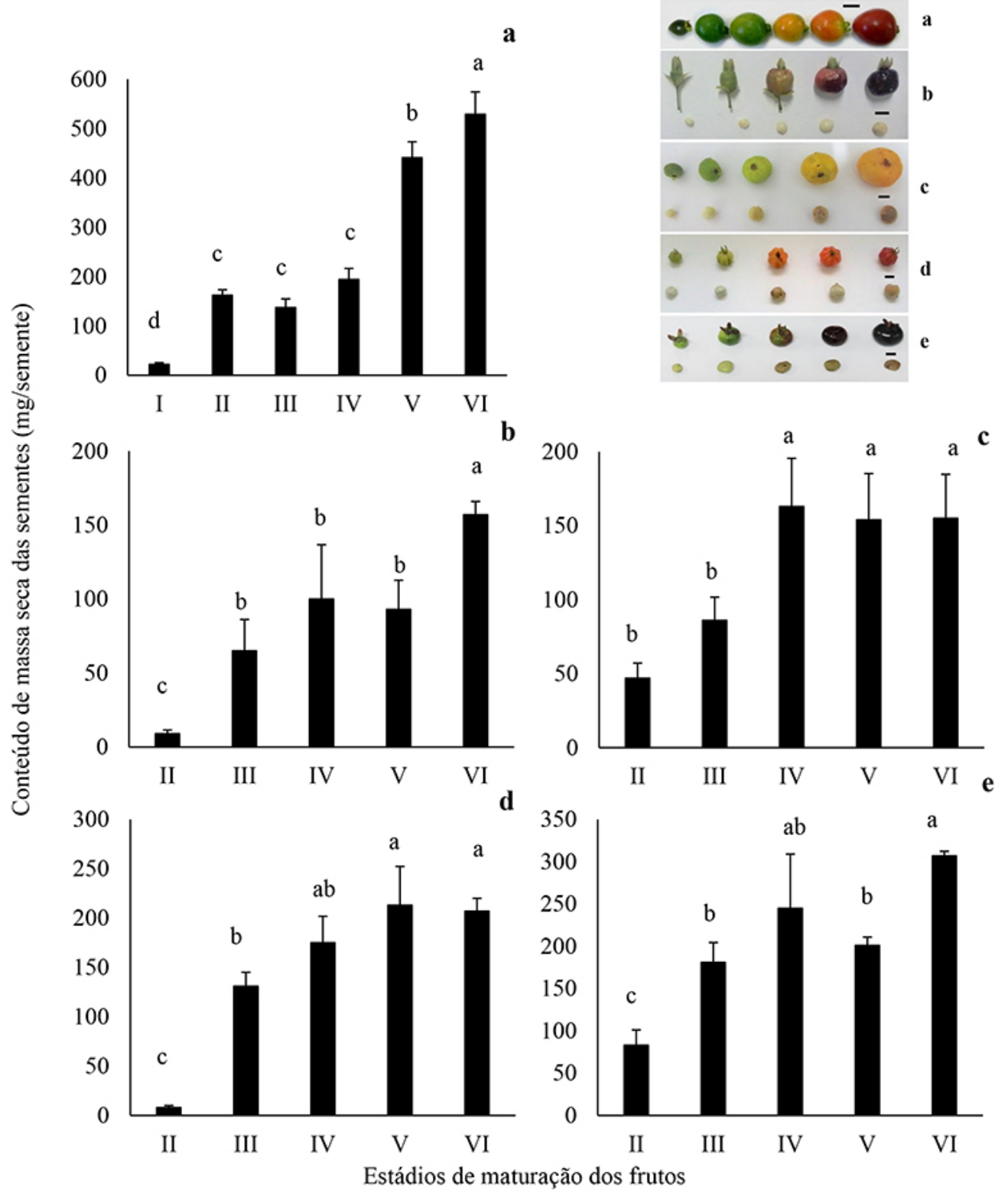

Figura 3. Conteúdo de massa seca (mg/semente) de sementes de espécies de Eugenia, em função do estádio de maturação dos frutos (I a VI), em função do estádio de maturação dos frutos (I a VI). a. Eugenia cerasiflora Miq. b. E. involucrata DC. c. E. pyriformis Cambess. d. E. uniflora L. e. E. brasiliensis Lam.). Médias seguidas pela mesma letra não diferem entre si pelo teste de Tukey (5\%).

Figure 3. Dry matter content (mg semente ${ }^{-1}$ ) of seeds of Eugenia species, according to the fruit maturity stages (I to VI). (a. Eugenia cerasiflora Miq. b. E. involucrata DC. c. E. pyriformis Cambess. d. E. uniflora L. e. E. brasiliensis Lam.). Means followed by the same letter do not differ by Tukey's test (5\%).

maturidade fisiológica da semente, com mudanças nas cores do fruto, sugere uma ligação com a síndrome de dispersão, uma vez que poderia atrair potenciais animais dispersores (Piña-Rodrigues \& Aguiar 1993). De fato, as sementes do último estádio são adequadas para dispersão e colonização, apresentando elevada qualidade fisiológica. Contudo, deve-se salientar que sementes de estádios mais imaturos também apresentaram essa capacidade, como as dos estádios IV e $\mathrm{V}$ de todas as espécies estudadas, $\operatorname{com} c a .50 \%$ ou mais de produção de plântulas.
Por outro lado, as sementes de E. cerasiflora e de $E$. involucrata acumularam massa seca até o último estádio (figura 3 a e b), mesmo não tendo redução do teor de água do estádio V para o VI (figura 2 a e b) e apresentando mais elevados valores de germinação e desenvolvimento de plântulas normais já no estádio $\mathrm{V}$ (figuras 4 a e c e 5 a e c), sugerindo um acúmulo de massa seca não essencial para a produção de plântulas.

A partir dos resultados obtidos neste trabalho nota-se a dificuldade em se definir o clássico ponto de maturidade 


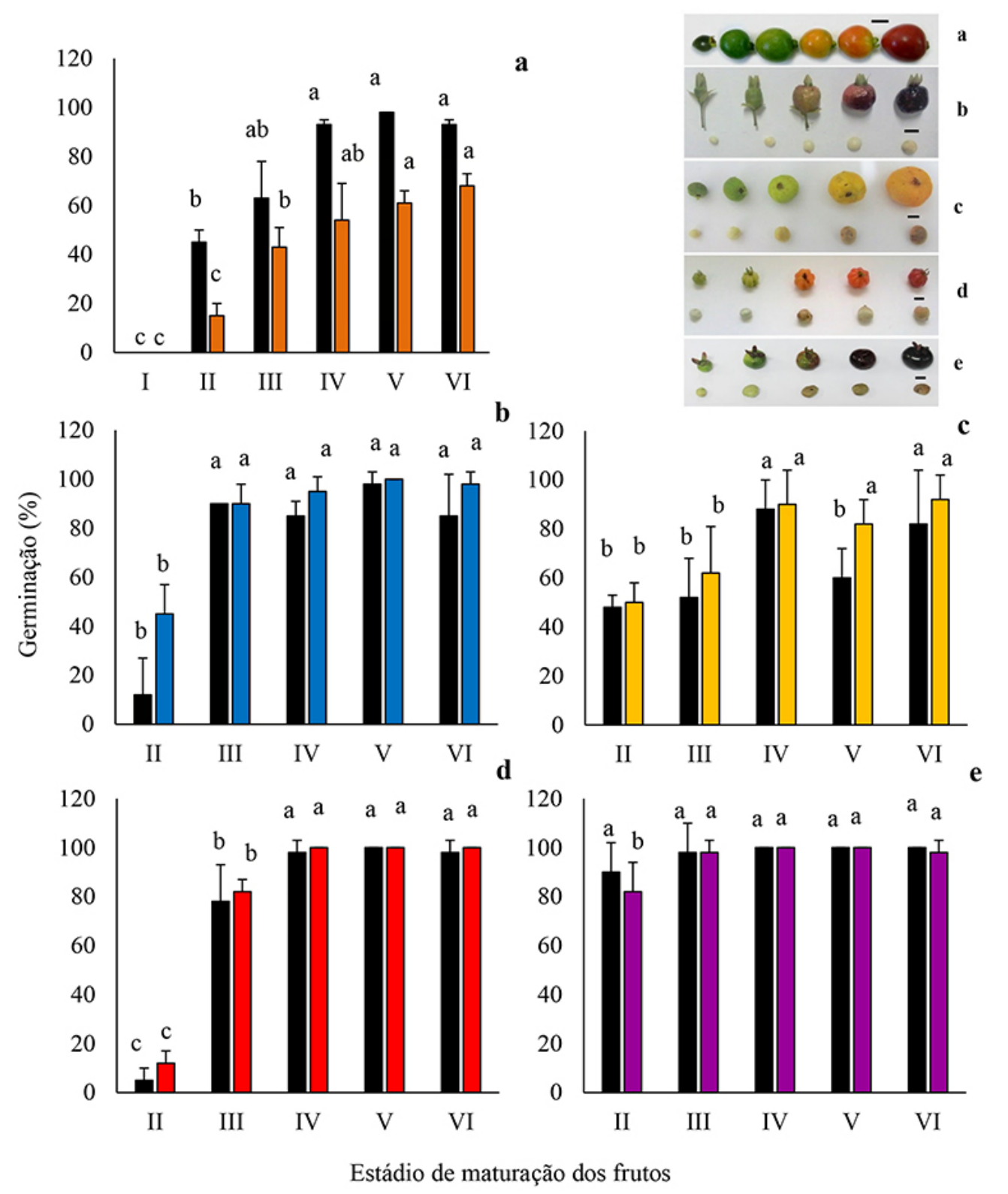

Figura 4. Germinação (\%) de sementes de espécies de Eugenia, em função do estádio de maturação dos frutos (I a VI). a. Eugenia cerasiflora Miq. b. E. involucrata DC. c. E. pyriformis Cambess. d. E. uniflora L. e. E. brasiliensis Lam.. Colunas pretas: sementes íntegras; coloridas: sementes com metade removida. Médias seguidas pela mesma letra, entre estádios de maturação, dentro de cada cor, não diferem entre si pelo teste de Tukey (5\%).

Figure 4. Germination (\%) of seeds of Eugenia species, according to the fruit maturity stages (I to VI). a. Eugenia cerasiflora Miq. b. E. involucrata DC. c. E. pyriformis Cambess. d. E. uniflora L. e. E. brasiliensis Lam.. Black columns: whole seeds; Colored columns: half seeds. Means followed by the same letter into the same color do not differ by Tukey's test (5\%).

fisiológica descrito para sementes ortodoxas ou mesmo estabelecer relação entre a maturação de frutos e sementes, para as espécies de Eugenia deste trabalho. Sementes provenientes de frutos verdes, totalmente imaturos, podem apresentar valores equivalentes de maturidade às de frutos maduros próximos à dispersão, como em E. brasiliensis. Também podem apresentar melhor equivalência entre frutos e sementes, como ocorreu com E. cerasiflora e $E$. involucrata. Contudo, embora as sementes de Eugenia apresentem capacidade de regenerar raízes e plântulas mesmo quando imaturas, segundo Teixeira \& Barbedo (2012) a eficiência dessa capacidade é maior nas maduras, provavelmente em função de uma maior quantidade de reservas nestas. Nas espécies deste trabalho, isso ocorreu 

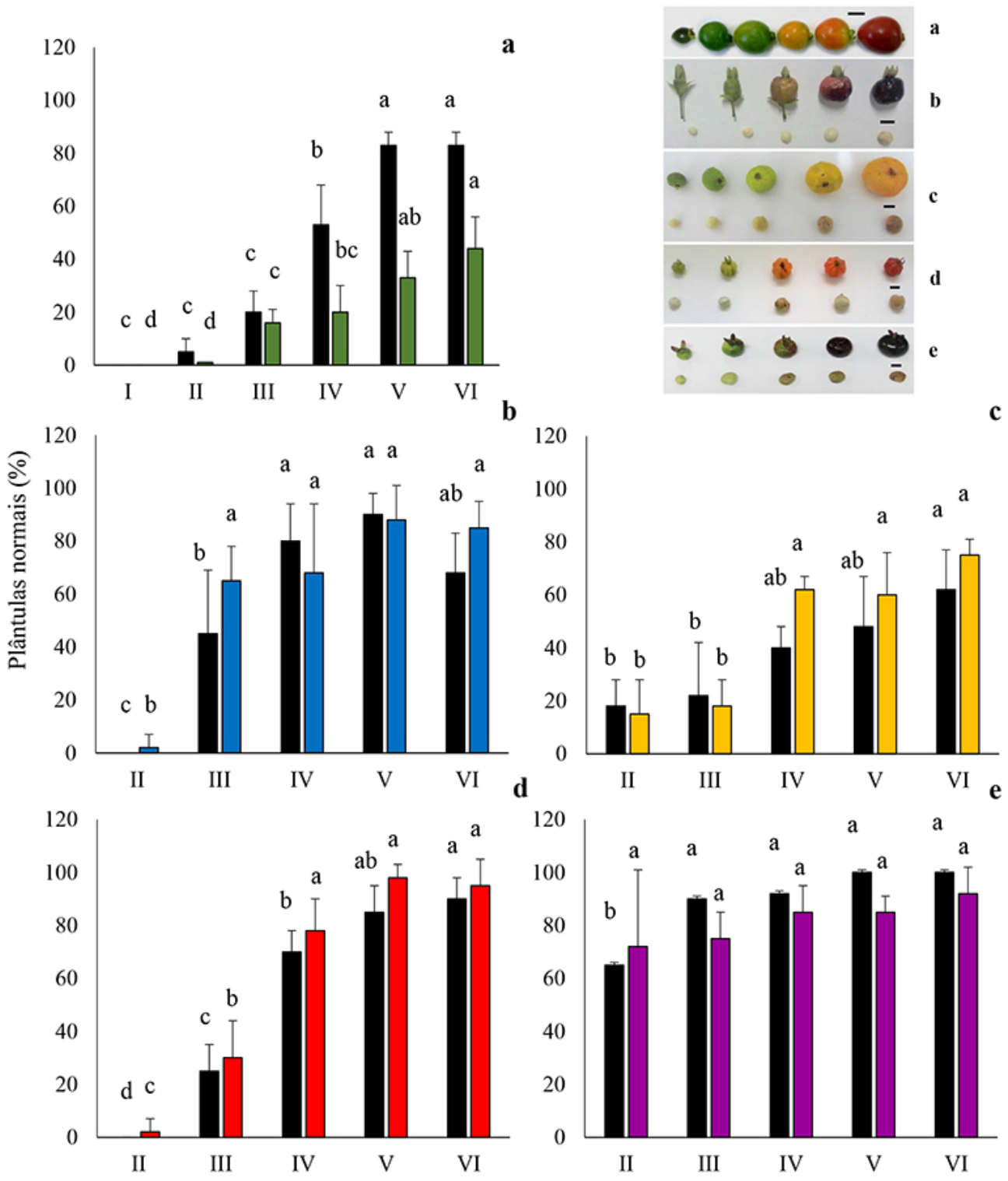

Estádios de maturação dos frutos

Figura 5. Desenvolvimento de plântulas normais de sementes de espécies de Eugenia, em função do estádio de maturação dos frutos (I a VI). a. Eugenia cerasiflora Miq. b. E. involucrata DC. c. E. pyriformis Cambess. d. E. uniflora L. e. E. brasiliensis Lam.. Colunas pretas: sementes íntegras; coloridas: sementes com metade removida. Médias seguidas pela mesma letra, entre estádios de maturação, dentro de cada cor, não diferem entre si pelo teste de Tukey (5\%).

Figure 5. Normal seedling development from seeds of Eugenia species, according to the fruit maturity stages (I to VI). a. Eugenia cerasiflora Miq. b. E. involucrata DC. c. E. pyriformis Cambess. d. E. uniflora L. e. E. brasiliensis Lam.. Black columns: whole seeds; Colored columns: half seeds. Means followed by the same letter into the same color do not differ by Tukey's test (5\%).

de forma progressiva para E. cerasiflora, até o estádio III para $E$. involucrata, até o estádio IV, para E. pyriformis e E. uniflora e praticamente não ocorreu para $E$. brasiliensis (figuras 4 e 5).
Os resultados de germinação e desenvolvimento de plântulas normais das sementes fracionadas demonstram que mesmo as sementes mais imaturas, oriundas de frutos em fases iniciais de desenvolvimento, já apresentam alguma 


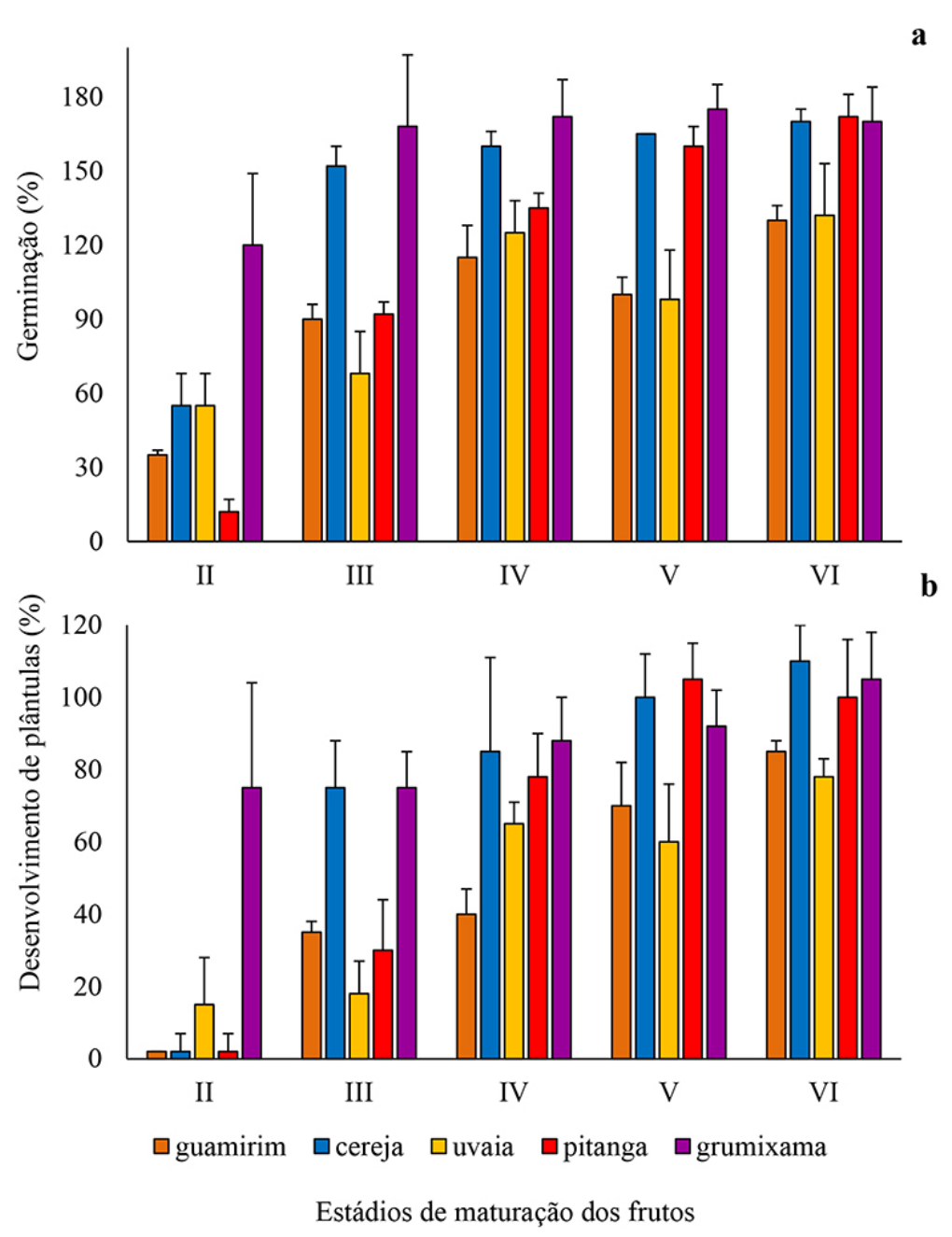

Figura 6. Germinação (a) e desenvolvimento de plantas (b) da soma das duas metades de sementes de Eugenia cerasiflora Miq. (guamirim), E. involucrata DC. (cereja), E. pyriformis Cambess. (uvaia), E. uniflora L. (pitanga) e E. brasiliensis Lam. (grumixama), em função do estádio de maturação dos frutos (I a VI).

Figure 6. Germination (a) and normal seedling development (b) from the sum of the two halves of the seeds of Eugenia cerasiflora Miq. (guamirim), E. involucrata DC. (cereja), E. pyriformis Cambess. (uvaia), E. uniflora L. (pitanga) e E. brasiliensis Lam. (grumixama), according to the fruit maturity stages (I to VI).

capacidade em produzir raízes (figura 6 a) mesmo não tendo acumulado sequer um terço da massa seca das sementes do último estádio (figura 3). Isso demonstra que nem toda a reserva acumulada nessas sementes é necessária para germinar. Em uma situação extrema, pode-se verificar, novamente para $E$. brasiliensis, que com apenas cerca de um quarto da massa seca final (figura 3 e) as sementes são capazes de produzir plântulas normais mesmo removendose metade do tecido de reserva (figura $5 \mathrm{e}$ ). Isso corrobora a estratégia descrita por Alonso et al. (2019) e Alonso \& Barbedo (2020) de que essas sementes investiram evolutivamente na germinação sequencial como forma de garantir a possibilidade de colonização por longos períodos, e não em bancos, no solo, de sementes tolerantes à dessecação com diferentes graus de dormência. Em outro extremo, E. cerasiflora foi a que apresentou os menores valores de germinação e plântulas normais quando metade do tecido de reserva é removido (figuras 4 e 5). Curiosamente, esta é, dentre todas as estudadas, a espécie com menor grau de domesticação, sendo menos conhecida pela população em geral e menos utilizada.

Os resultados de germinação e desenvolvimento de plântulas normais da soma das frações (figura 6) demonstram que o potencial de regeneração de raízes não garante o desenvolvimento de plântulas. Segundo Teixeira \& Barbedo (2012), isso pode se dever ao fato de a semente fracionada perder reservas importantes. Contudo, a capacidade de ao menos iniciar o desenvolvimento de uma nova planta, 
pela formação de raiz, mesmo em sementes de frutos imaturos, revela a grande probabilidade de que a semente germine e produza uma nova planta. Também deve ser levado em conta o fato de que muitos animais se alimentam desses frutos, entre eles os dispersores, principalmente pássaros, e que podem danificar apenas parcialmente a semente. Diferentemente do que foi realizado no presente trabalho, em que se removeu metade da semente, pequenas remoções poderiam permitir o desenvolvimento completo de uma nova planta, como demonstrado anteriormente em sementes de E. pyriformis (Silva et al. 2003). Esta capacidade regenerativa, demonstrada anteriormente em sementes de frutos maduros (Anjos \& Ferraz 1999, Silva et al. 2003 e Silva et al. 2005), neste trabalho foi observada até em sementes de frutos totalmente imaturos, sugerindo a presença de células totipotentes, que promovem a formação de embriões somáticos a partir do embrião zigótico, já em sementes imaturas.

Em relação ao amadurecimento de sementes e frutos, outras espécies de Myrtaceae também apresentaram ausência de sincronismo entre ambos, como Calyptranthes clusiifolia (Miq.) O. Berg. (Souza-Junior 2007). Também há recomendação, por alguns autores, de colheita antes do final da maturação dos frutos, como forma de evitar ataques de insetos (Hirano \& Possamai 2008). O teor de água das sementes, embora seja amplamente utilizado como indicador de maturação, não pode ser padronizado para as de Eugenia, talvez justamente por não haver separação entre as fases de formação e germinação (Barbedo 2018). Algumas sementes podem ter atingido máximo desenvolvimento e maturação e, por algum tempo depois, terem sido armazenadas dentro dos frutos até a dispersão destes. Muitos estudos feitos com maturação de sementes de diversas espécies apontam o ponto de máximo conteúdo de matéria seca como o melhor e mais seguro indicativo de que as sementes atingiram a maturidade fisiológica (Araujo et al. 2006). Porém, a resposta distinta apresentada pelas cinco espécies estudadas, para essa característica, também não permite estabelecer qualquer padrão de comportamento para o gênero. Independente da possibilidade ou não de um correto diagnóstico do momento ideal de colheita, a partir dos resultados deste trabalho ficou evidente que sementes das cinco espécies possuem alta capacidade de germinar e produzir plântulas normais mesmo quando obtidas de frutos imaturos e com perda de material de reserva.

\section{Agradecimentos}

Os autores agradecem ao Instituto de Botânica, pela permissão para as coletas e ao Programa de PósGraduação em Biodiversidade Vegetal e Meio Ambiente, pela oportunidade dada à primeira Autora para o curso de Doutorado e execução da Tese, da qual este trabalho faz parte.

\section{Literatura citada}

Alonso C.R. \& Barbedo C.J. 2020. Germinações sucessivas em sementes de Eugenia spp. Hoehnea 47: no prelo.

Alonso C.R., Asperti L.M., Guardia M.C. \& Barbedo C.J. 2019. Cutting and regeneration of roots and seedlings from seeds of Eugenia candolleana DC. at different maturity. Journal of Seed Science 41: 160-167.

Amador T.S. \& Barbedo C.J. 2011. Potencial de inibição da regeneração de raízes e plântulas em sementes germinadas de Eugenia pyriformis. Pesquisa Agropecuária Brasileira 46: 814-821.

Amador T.S. \& Barbedo C.J. 2015. Germination inhibits the growth of new roots and seedlings in Eugenia uniflora and Eugenia brasiliensis. Journal of Seed Science 37: 241-247.

Andrade, A.C.S., Cunha, R., Souza, A.F., Reis, R.B. \& Almeida, K.L. 2003. Physiological and morphological aspects of seed viability of a neotropical savannah tree, Eugenia dysenterica DC. Seed Science and Tecnology 31: 125-137.

Andrade. R.N.B. \& Ferreira, A.G. 2000. Germinação e armazenamento de sementes de uvaia (Eugenia pyriformis Camb.) - Myrtaceae. Revista Brasileira de Sementes 22: 118-125.

Anjos, A.M.G. \& Ferraz, I.D.K. 1999. Morfologia, germinação e teor de água das sementes de araçá-boi (Eugenia stipitata ssp. sororia). Acta Amazônica 29: 337-348.

Araujo, E.F., Araujo, R.F., Sofiatti, V. \& Silva, R.F. 2006. Maturação de sementes de milho-doce - grupo super doce. Revista Brasileira de Sementes 28: 69-76.

Avila, A.L., Argenta, M.S., Muniz, M.F.B., Poleto, I. \& Blumes, E. 2009. Maturação físiológica e coleta de sementes de Eugenia uniflora L. (pitanga). Ciência Florestal 19: 61-68.

Barbedo, C.J. 2018. A new approach towards the so-called recalcitrant seeds. Journal of Seed Science 40: 221-236.

Barbedo, C.J. \& Santos Junior, N.A. 2018. Sementes do Brasil: produção e tecnologia para espécies da flora brasileira. Instituto de Botânica, São Paulo.

Barbedo C.J., Centeno D.C. \& Figueiredo-Ribeiro R.C.L. 2013. Do recalcitrant seeds really exist? Hoehnea 40: 583-593.

Brasil. 2009. Regras para Análise de Sementes. Ministério da Agricultura, Pecuária e Abastecimento, Brasília.

Danner, M.A., Citadin, I., Sasso, S.A.Z., Sachet, M.R. \& Ambrósio, R. 2010. Fenologia da floração e frutificação de mirtáceas nativas da floresta com araucária. Revista Brasileira de Fruticultura 32: 291-295.

Delgado, L.F. 2010. Fracionamento, maturação e origem da capacidade regenerativa de sementes de algumas espécies brasileiras de Eugenia (Myrtaceae). Tese de Doutorado, Instituto de Botânica São Paulo. 
Delgado, L.F. \& Barbedo, C.J. 2007. Tolerância à dessecação de sementes de espécies de Eugenia. Pesquisa Agropecuária Brasileira 42: 265-272.

Delgado L.F. \& Barbedo C.J. 2011. Atividade inibidora da germinação em extratos de sementes de Eugenia uniflora L. Revista Brasileira de Sementes 33: 463-471.

Delgado L.F., Mello J.I.O. \& Barbedo C.J. 2010. Potential for regeneration and propagation from cut seeds of Eugenia (Myrtaceae) tropical tree species. Seed Science and Technology 38: 624-634.

Fidalgo A.O., Cécel A.T., Mazzi J.F.O. \& Barbedo C.J. 2019. Pollination and quality of seeds and plantlets of Eugenia uniflora L. Hoehnea 46: e052018.

Guardia, M.C., Asperti, L.M., Fidalgo, A.O. \& Garcia, V.A. 2018. Produção e obtenção de sementes. In: C.J. Barbedo \& N.A. Santos Junior (orgs.). Sementes do Brasil: produção e tecnologia para espécies da flora brasileira. São Paulo: Instituto de Botânica, pp. 41-62.

Hirano, E. \& Possamai, E. 2008. Estádio de maturação do fruto e germinação de sementes de três espécies de Lauraceae. Scientia Agraria 9: 219-223.

Inocente M.C. \& Barbedo C.J. 2019. Germination of Eugenia brasiliensis, E. involucrata, E. pyriformis, and E. uniflora (Myrtaceae) under water-deficit conditions. Journal of Seed Science 41: 76-085.

Lamarca E.V., Prataviera J.S., Borges I.F., Delgado L.F., Teixeira C.C., Camargo M.B.P., Faria J.M.R. \& Barbedo, C.J. 2013. Maturation of Eugenia pyriformis seeds under different hydric and thermal conditions. Anais da Academia Brasileira de Ciências 85: 223-233.

Lamarca E.V., Camargo M.B.P., Teixeira, S.P., Silva, E.A.A. Faria J.M.R. \& Barbedo, C.J. 2016. Variations in desiccation tolerance in seeds of Eugenia pyriformis: dispersal at different stages of maturation. Revista Ciência Agronômica 47: 118-126.
Marcos-Filho, J. 2015. Fisiologia de sementes de plantas cultivadas. ABRATES, Londrina.

Piña-Rodrigues, F.C.M. \& Aguiar, I.B. 1993. Maturação e Dispersão de Sementes. In: Sementes florestais tropicais (Aguiar, I.B., Piña-Rodrigues, F.C.M. \& Figliolia, M.B., Coord.). Brasília, Abrates, pp. 215-174.

Prataviera J.S., Lamarca E.V., Teixeira C.C. \& Barbedo, C.J. 2015. The germination success of the cut seeds of Eugenia pyriformis depends on their size and origin. Journal of Seed Science 37: 47-54.

Santos, C.M.R., Ferreira, A.G. \& Áquila, M.E.A. 2004. Características de frutos e germinação de sementes de seis espécies de Myrtaceae nativas do Rio Grande do Sul. Ciência Florestal 14: 13-20.

Silva, C.V., Bilia, D.A.C. \& Barbedo, C.J. 2005. Fracionamento e germinação de sementes de Eugenia. Revista Brasileira de Sementes 27: 86-92.

Silva, C.V.; Bilia, A.C., Maluf, A.M. \& Barbedo, C.J. 2003. Fracionamento e germinação de sementes de uvaia (Eugenia pyriformis Cambess. - Myrtaceae ). Revista Brasileira de Botânica 26: 213-221.

Silva, E.E.A., Oliveira, J.M. \& Pereira, W.V.S. 2018. Fisiologia das sementes. In: C.J. Barbedo \& N.A. Santos Junior (orgs.). Sementes do Brasil: produção e tecnologia para espécies da flora brasileira. São Paulo: Instituto de Botânica, pp. 15-40.

Souza-Júnior, C.N., Bernardo, V., Barbosa, J.M., Castan, G.S. \& Menegucci, Z.R.H. 2007. Coloração dos Frutos como Indicador de Maturação de Sementes de Araçarana (Calypthrantes clusiifolia (Miq.) O. Berg). Revista Brasileira de Biociências 5(supl.2): 1131-1133.

Teixeira C.C. \& Barbedo C.J. 2012. The development of seedlings from fragments of monoembryonic seeds as an important survival strategy for Eugenia (Myrtaceae) tree species. Trees, Structure and Function 26: 1069-1077. 